\title{
Clinical Study Status
}

National Cancer Institute

\section{Source}

National Cancer Institute. Clinical Study Status. NCI Thesaurus. Code C70703.

A business process state of a clinical study in relation to its approval status, authorization for patient enrollment, and protocol-specific activities. 